\title{
INFLUENCIAS DEL COLOR DE FONDO SOBRE EL PROCESAMIENTO DE PATRONES VISUALES JERÁRQUICOS EN SUJETOS DISLÉXICOS, MALOS LECTORES Y NORMOLECTORES
}

\section{BACKGROUND COLOR INFLUENCES ON PROCESSING VISUAL HIERARCHICAL PATTERNS IN DYSLEXICS, POOR READERS AND NORMAL READERS.}

\author{
JOAQUín VIDAL, DOLORES LUNA Y MARÍA R. ElOSÚA* \\ Departamento de Psicología Básica I. Facultad de Psicología (UNED)
}

Recibido 10-02-04

Aceptado 20-04-04

\section{Resumen}

El objetivo de este trabajo consistió en comprobar si los sujetos disléxicos muestran un déficit en el procesamiento de la información global en patrones organizados jerárquicamente, que está mediado por el sistema magnocelular. Además, se analizó si el color de fondo (rojo y verde) afecta de manera diferente al procesamiento de la información global en lectores normales, malos lectores y sujetos disléxicos. Los resultados muestran que el color de fondo parece deteriorar la precisión en el procesamiento global de los patrones jerárquicos, pero este deterioro se da en todos los grupos por igual. Los resultados no apoyan la teoría que defiende la existencia de un déficit en el sistema de procesamiento magnocelular en sujetos que presentan problemas de lectura.

\section{Palabras elave}

Dislexia, color de fondo, procesamiento visual, patrones jerárquicos, y sistema magnocelular.

\begin{abstract}
The aim of this work was to examine whether dyslexia is associated to a deficit in the processing of global information of hierarchical patterns which is mediated by the magnocelular system. Moreover, effects of color background on global information processing are tested in normal readers, poor readers and dyslexics. The results showed that red background color impaired global processing in all three groups (normal readers, poor readers and dyslexics). These results are not congruent with the theory that proposes one deficit in the magnocellular processing system in disabled readers.
\end{abstract}

\section{Key words}

Dyslexia, background color, visual processing, hierarchical patterns, intelligence levels and magnocellular system.

\footnotetext{
* Este trabajo formó parte del trabajo presentado para la obtención del Diploma de estudios avanzados en el Programa de Doctorado del Departamento de Psicología Básica I. Facultad de Psicología. UNED
} 


\section{Introduceión}

La dislexia se define como una dificultad en la adquisición de las habilidades implicadas en la lectura, a pesar de una inteligencia normal, en ausencia de problemas físicos o psicológicos y con una escolarización adecuada (APA, 1994). Los sujetos disléxicos se diferencian de los malos lectores en que estos últimos no cumplen todos los criterios para ser clasificados como disléxicos debido a que sus problemas de lectura pueden provenir de una inadecuada. inteligencia, escolarización, motivación, etcétera. (Snow, Burns y Griffin, 1998). Sin embargo, la distinción entre ambos trastornos en ocasiones resulta difícil y es una cuestión de grado. La dislexia la padece entre un 4 y un $10 \%$ de la población aproximadamente (Rutter, 1978) y existe un amplio consenso de que se trata de un trastorno neurológico con un origen genético, pero todavía no se conocen con exactitud las bases cognitivas y neurológicas de este desorden (Ramus, 2001). Aunque las causas pueden ser diversas, la mayoría de los disléxicos presentan dificultades en el procesamiento fonológico (Ramus, 2001; Tallal, 1980) y algunos autores han encontrado también problemas en el procesamiento visual de estos sujetos, (Livingstone, Rosen, Drislane y Galaburda, 1991; Lovegrove, 1991) y han sugerido un déficit en el funcionamiento del sistema magnocelular.

En los últimos años se ha estudiado la relación entre los sistemas magno y parvocelular y el efecto de precedencia global. El primer estudio sobre precedencia global fue desarrollado por Navon (1977). Este efecto se consigue cuando presentamos a los sujetos patrones jerárquicos, que consisten en letras o símbolos grandes (nivel de procesamiento global) formados por letras o símbolos pequeños (nivel de procesamiento local). Este autor comprobó que al realizar estas presentaciones se producía siempre una superioridad (primacía o precedencia) del procesamiento global que se podía cuantificar de dos maneras. Por una parte, se daba una ventaja global, es decir, los estímulos globales se nombraban antes y producían menos errores al ser nombrados que los estímulos locales. Por otra parte, se observaba un efecto de interferencia que consistía en que se tardaba menos tiempo en nombrar y se cometían menos errores, con los estímulos congruentes (estímulos globa- les y locales similares) que con los estímulos incongruentes (estímulos globales y locales diferentes).

Michimata, Okubo y Mugishima (1999) han analizado los efectos de la modificación del funcionamiento del sistema magnocelular sobre el efecto de precedencia global en sujetos normales variando el funcionamiento del sistema magnocelular en una tarea de atención selectiva al nivel global y local para examinar el efecto de precedencia global. Los trabajos de investigaciones anteriores (Livingstone y Hubel, 1984; Marrocco, McClurkin y Young, 1988) habían demostrado que el sistema magnocelular contenía células $M$ Tipo IV, y estas células disminuían su actividad cuando estaba presente la luz roja. Además, presentando los estímulos sobre un fondo de color rojo, empeoró la ejecución en tareas que requerían procesamiento de las frecuencias espaciales bajas (Breitmeyer y Breier, 1994) y frecuencias temporales altas (Breitmeyer y Williams, 1990). Los resultados de estos trabajos sugieren que la manipulación del color de fondo se podría utilizar para variar el funcionamiento del sistema magnocelular. A partir de estos primeros estudios, Michimata, Okubo y Mugishima (1999) utilizaron una tarea de atención selectiva a la información global y local y comprobaron que cuando se utilizaban fondos verdes se observaba un efecto de una interferencia global asimétrica (el resultado obtenido en este tipo de estudios), mientras que los fondos rojos atenuaban el efecto de primacía global y y se observanba un efecto de interferencia simétrica entre los niveles global y local. Estos autores concluyeron que el sistema magnocelular humano era responsable, al menos en parte, del efecto de precedencia global.

Hasta la fecha no se ha examinado la ejecución de los sujetos disléxicos en tareas de atención selectiva a la información global y local. Sin embargo, algunos trabajos han estudiado la ejecución de sujetos disléxicos en tareas en las que se ha manipulado la organización perceptiva que media el efecto de precedencia global (Han y Humphreys, 1999) como el agrupamiento perceptivo (Lewis y Frick, 1999), y la integración de contornos (Simmers y Bex, 2001). Por un lado, Lewis y Frick (1999) seleccionaron sujetos que presentaron dificultades 
para realizar agrupamiento perceptivo de filas formadas por símbolos, por ejemplo, " $\mathrm{O}$ " $\mathrm{y}$ " $\mathrm{X}$ ", y encontraron que el $60 \%$ de los sujetos que presentaron esta ceguera a las filas tenía problemas relacionados con la dislexia. Sin embargo, los mismos autores comentaron que utilizaron el término dislexia en sentido amplio para referirse a sujetos con dificultades en el procesamiento del lenguaje escrito. Es decir, la selección de sujetos fue diferente a como se suele realizar normalmente. En la mayoría de las investigaciones se seleccionan sujetos con dislexia o con otros problemas de lectura y se someten a prueba las hipótesis que se han planteado previamente. Sin embargo, en su trabajo, se seleccionaron sujetos con ceguera a las filas ("row blindness") y se vio si tenían dislexia, sin controlar la variable inteligencia de los sujetos. Como indicaron los mismos autores, "es probable que se diera un sesgo al alza en la proporción de disléxicos con ausencia de agrupamiento". Es decir, al utilizar la clasificación de los sujetos disléxicos en sentido amplio y no tener en cuenta los niveles de inteligencia de cada sujeto, pudieron ser catalogados como sujetos disléxicos muchos sujetos malos lectores.

Por otra parte, Simmers y Bex (2001) utilizaron una tarea de detección de contornos (patrones globales), a partir de pequeños patrones de ondas Gabor (estímulos locales). Sus resultados indicaron que los sujetos disléxicos cometían significativamente más errores que los sujetos de control al detectar contornos (patrones globales), a partir de estímulos locales. Sin embargo, este trabajo también presentó algunas limitaciones metodológicas que se van a mencionar. En primer lugar, estos autores definieron a los disléxicos como: "sujetos con una edad de lectura menor en una desviación típica a la esperada según su nivel de inteligencia", por lo que tampoco se hizo distinción entre malos lectores y sujetos disléxicos. Además, para seleccionar a los sujetos de control únicamente tuvieron en cuenta que "no informaran de haber tenido problemas de lectura". Es decir, no se midió ni su nivel de lectura ni su nivel de inteligencia. En segundo lugar, no se registró el tiempo de respuesta, por lo que pudo haber intercambio entre la velocidad y la precisión de respuesta.

Aunque no existe un acuerdo generalizado sobre la manera en que la vía $\mathrm{M}$ (magnocelular) puede afectar a la lectura (Skottun, 1997, 2000), se han desarrollado dos modelos para analizar este problema: Según el modelo de Breitmeyer (1980), cualquier texto sería procesado únicamente por la vía $\mathbf{P}$ (parvocelular) y la función de la vía $M$ sería la de inhibir la actividad de la vía $P$ mientras se realizan los movimientos sacádicos, suprimiendo la persistencia visual y evitando que la imagen se haga borrosa como consecuencia de la superposición de varias imágenes procedentes de varias fijaciones. Sin embargo, este modelo ha sido criticado porque las investigaciones psicofisiológicas posteriores (Skottun, 2000; Skottun y Parke, 1999) parecen demostrar que la supresión visual sacádica elimina la actividad del sistema magnocelular, mientras que no afecta a la actividad del sistema parvocelular. En el segundo modelo propuesto, Chase (1996) supuso que durante el procesamiento visual inicial, llegaría, en primer lugar, la información con las bajas frecuencias espaciales proviniente de la vía magnocelular, mientras que en las etapas posteriores, las altas frecuencias espaciales se añadirían a la información preexistente y proporcionarían información sobre los detalles finos. Siguiendo este modelo (Chase, Ashourzadeh, Kelly, Monfette y Kinsey, 2003), la identificación perceptual se formaría inmediatamente después de que llegara la información sobre las bajas frecuencias espaciales, y a continuación se integraría la información sobre los detalles (altas frecuencias espaciales) en la representación mental inicial. Si se dispusiera de suficiente información, las palabras serían identificadas rápidamente a partir de la información de la vía magnocelular. Sin embargo, cuando el sistema ortográfico no pudiera realizar la identificación, el sistema debería esperar la información más detallada de la vía parvocelular. Si la vía magnocelular procesara información relacionada con los textos escritos, la ejecución durante la lectura se podría ver afectada por algunos factores visuales que cambiaran el funcionamiento de las células del sistema magnocelular (Chase et al., 2003). Para poner a prueba este modelo, estos autores estudiaron mediante cuatro experimentos, los efectos del color de fondo sobre la modificación de la intensidad de funcionamiento del sistema magnocelular durante la lectura. En los tres primeros experimentos utilizaron niveles normales de contraste de los textos escritos y encontraron que en lugar de producirse una mejora con los filtros azules como 
habían propuesto algunos autores (Iovino, Fletcher, Breitmeyer y Foorman, 1998; Solan, 1998), se producía más bien un empeoramiento con los filtros rojos. Según estos autores, en condiciones normales de contraste, el sistema magnocelular estaría funcionando correctamente y al añadir luz roja se deterioraría el funcionamiento de este sistema, produciendo un empeoramiento de la lectura. Sin embargo, en el cuarto experimento, se potenció el funcionamiento de la vía parvocelular haciendo que la luminancia del texto y el fondo fueran las mismas. En estas condiciones, los sujetos mejoraron su rendimiento al utilizar filtros de color rojo. Por lo tanto, según estos autores, la luz roja empeora la lectura cuando el sistema magnocelular funciona en condiciones normales, mientras que mejora la velocidad de la lectura cuando se potencia el funcionamiento del sistema parvocelular.

Teniendo en cuenta los estudios realizados hasta el momento, el primer objetivo de la presente investigación consistió en examinar si los sujetos disléxicos mostraban un déficit en la ejecución de una tarea de atención selectiva a la información global en patrones organizados jerárquicamente. Ya que el efecto de precedencia global parece estar mediado por el sistema magnocelular, si los sujetos disléxicos presentan un déficit en el funcionamiento de este sistema presentarían peor ejecución en el procesamiento global que en el local, en comparación con los otros grupos que deberían presentar el efecto de precedencia global. El segundo objetivo consistió en examinar si el color de fondo afectaba de manera diferente al procesamiento de la información global y si el deterioro era diferente en los sujetos normolectores en comparación con los malos lectores y disléxicos. Si esto es así, la ejecución bajo la condición de color de fondo rojo empeoraría la ejecución en la tarea de atención selectiva al nivel global, en comparación con la condición de color de fondo verde y el deterioro en la ejecución sería más pronunciado en sujetos malos lectores y disléxicos que en sujetos normolectores.

\section{Método}

\section{Participantes}

A partir de una muestra de 202 alumnos de educación secundaria obligatoria, se selec- cionaron 3 grupos (lectores normales, malos lectores y disléxicos) de 16 sujetos cada uno, con diferentes puntuaciones en inteligencia general y en inteligencia verbal (Yuste, 1995), de manera que los grupos de normolectores y disléxicos se diferenciaron de manera significativa, en las puntuaciones de inteligencia general (IG), del grupo de malos lectores (comparación de las puntuaciones en IG entre los grupos de: normolectores-malos lectores $\mathrm{t}=8.70, \mathrm{p}<0.001 \mathrm{y}$ disléxicos-malos lectores $\mathrm{t}=4.62, \mathrm{p}<0.001$ ), mientras que los grupos de malos lectores y disléxicos se diferenciaron de manera significativa, en las puntuaciones de inteligencia verbal (IV), del grupo de lectores normales (comparación de las puntuaciones en IV entre los grupos de: normolectores-malos lectores $t=6.74$, $\mathrm{p}<0.001$ y normolectores-disléxicos $\mathrm{t}=6.61$, $\mathrm{p}<0.001$, respectivamente). El nivel de lectura se determinó mediante la prueba PROLEC-SE (Ramos y Cuetos, 1999).

\section{Estímulos y aparatos}

Se presentaron como estímulos letras $\mathrm{C}$ y $\mathrm{U}$ grandes formadas por letras $\mathrm{C}$ y $U$ pequeñas (véase la Figura 1).

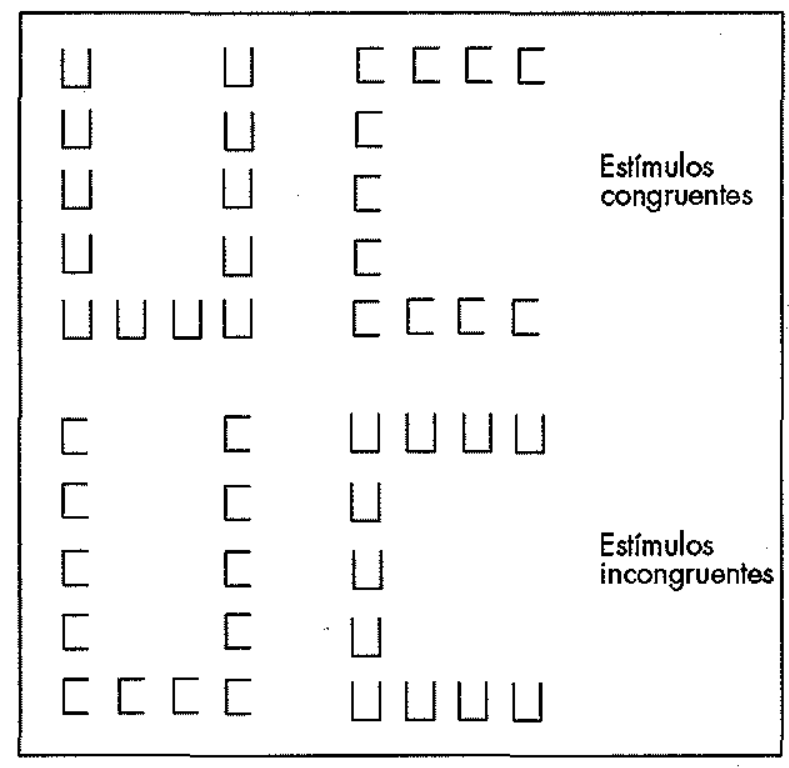

Figura 1. Estímulos presentados a los sujetos. Los estímulos cuyas letras global y local son las mismas se denominan congruentes, mientras que los que tienen letras global y local diferentes se denominan incongruentes 
Los estímulos se mostraron en un ordenador personal, modelo Authentic AMD-K6 (tm) 3D processor, con pantalla Philips de 15 pulgadas.

Las características de los estímulos fueron las siguientes: el tamaño de la letra global fue de $33 \mathrm{~mm}$ de alto y $24 \mathrm{~mm}$ de ancho $\left(3.78^{\circ}\right.$ y $2.63^{\circ}$ respectivamente de ángulo visual para una distancia de $50 \mathrm{~cm}$ ); b) el tamaño de letra local fue de $4 \mathrm{~mm}$ de alto y $3 \mathrm{~mm}$ de ancho $\left(0.46^{\circ}\right.$ y $0.34^{\circ}$ de ángulo visual); c) las letras de color negro se presentaron sobre un fondo de color verde o rojo, igualados en luminancia (5.4 $\mathrm{cd} / \mathrm{m} 2$ ) y d) la separación entre letras horizontales fue de $4 \mathrm{~mm}\left(0.46^{\circ}\right)$ y para las verticales fue de $3 \mathrm{~mm}\left(0.34^{\circ}\right)$.

Las letras globales se formaron colocando las letras locales en una matriz de 5 (vertical) $\mathrm{x}$ 4 (horizontal). Antes de que apareciera cada estímulo, se presentó un punto de fijación en el centro de la pantalla, que consistió en una cruz de $4 \mathrm{~mm}$ de alto por $3 \mathrm{~mm}$ de ancho $\left(0.46^{\circ} \mathrm{x}\right.$ $0.34^{\circ}$ de ángulo visual), e inmediatamente después de cada estímulo se presentó una máscara formada por trazos negros distribuidos al azar por toda la pantalla.

\section{Diseño y procedimiento}

El diseño experimental incluyó tres factores: a) condiciones de matiz de fondo: verde y rojo (estímulos con la misma luminancia y saturación, pero con un matiz diferente); b) condiciones de atención: global y local (el sujeto debía dirigir su atención hacia la letra global o hacia la letra local); c) condiciones de congruencia: si la letra global era la misma que la letra local el estímulo se consideraba congruente y si la letra global era diferente que la letra local el estímulo era incongruente; y d) condiciones de letra presentada (se utilizaron letras $\mathrm{C}$ y $\mathrm{U}$ ).

Se consideró como factor intersujetos el grupo al que pertenecían los sujetos (lectores normales, malos lectores y disléxicos). Las condiciones de matiz se presentaron en dos bloques distintos de ensayos. Las condiciones de atención se presentaron separadas en dos partes, en la primera mitad se pedía a los sujetos que dirigieran la atención a los estímulos globales y en la segunda mitad del bloque se les pedía que dirigieran la atención hacia los estímulos locales. Las condiciones de congruencia se distribuyeron de manera aleatoria dentro de cada bloque. Los bloques estaban formados por 160 ensayos experimentales, más 8 ensayos de práctica. Se proporcionó información de los errores y aciertos únicamente en los ensayos de práctica. El orden de los ensayos y las condiciones de atención fueron contrabalanceadas entre los sujetos de cada grupo. La tarea de los sujetos consistía en presionar una tecla del ordenador para la letra $\mathrm{C}$ y otra tecla diferente para la letra U en cada una de las condiciones de atención (global y local).

La secuencia experimental consistió en la presentación de un punto de fijación en el centro de la pantalla durante $500 \mathrm{~ms}$, a continuación aparecía el estímulo durante $150 \mathrm{~ms}$ y la presentación terminaba con una máscara que duraba $150 \mathrm{~ms}$. Se pidió a todos los sujetos que respondieran lo más rápidamente posible y que intentaran no cometer errores.

\section{Resultados}

Los tiempos de reacción (TRs) y las tasas de error se analizaron por separado mediante dos ANOVAs de medidas repetidas en cuatro factores: color de fondo (rojo y verde), condiciones de atención (global y local), congruencia (estímulos congruentes e incongruentes), y letras (C y U). Los TRs inferiores a 200 milisegundos y superiores a 1500 se eliminaron del análisis, así como aquellos correspondientes a las respuestas incorrectas. Las medias correspondientes a los TRs y los porcentajes de error para todas las condiciones experimentales se presentan en la Tabla 1. No se observó intercambio entre velocidad y precisión $(r=+0.14)$.

En el análisis de los tiempos de respuesta resultaron significativos los efectos principales de congruencia, $\mathrm{F}(1,33)=38.58, \mathrm{MCE}=86418.61$, $\mathrm{p}<0.001$ y letra $\mathrm{F}(1,33)=6.52, \mathrm{MCE}=39210.11$, $\mathrm{p}=0.02$; sin embargo, los resultados más relevantes se obtuvieron al analizar las tasas de error, por lo que se comentan a continuación.

En el análisis de los errores resultó significativo el efecto principal de congruencia, $F(1,33)=17.53, M C E=966.17, p<0.001$, que indicó que los estímulos congruentes producían 
Tabla 1. Tiempos de reacción medios ( $y$ tasas de error) en todas las condiciones experimentales

\begin{tabular}{lccccccccc}
\hline & \multicolumn{8}{c}{ Global } & \multicolumn{7}{c}{ Local } \\
\cline { 2 - 9 } & \multicolumn{1}{c}{ Congruente } & \multicolumn{2}{c}{ Incongruente } & \multicolumn{2}{c}{ Congruente } & \multicolumn{2}{c}{ Incongruente } \\
\cline { 2 - 9 } & $\mathrm{C}$ & $\mathrm{U}$ & $\mathrm{C}$ & $\mathrm{U}$ & $\mathrm{C}$ & $\mathrm{U}$ & $\mathrm{C}$ & $\mathrm{U}$ \\
\cline { 2 - 9 } & 660 & 645 & 686 & 680 & 681 & 648 & 703 & 667 \\
Lectores & $(3.83)$ & $(4.83)$ & $(6.08)$ & $(6.00)$ & $(1.67)$ & $(3.33)$ & $(3.75)$ & $(2.92)$ \\
normales & 666 & 622 & 648 & 670 & 714 & 669 & 715 & 672 \\
Malos & $(9.42)$ & $(6.33)$ & $(17.58)$ & $(15.25)$ & $(5.33)$ & $(4.67)$ & $(8.67)$ & $(6.17)$ \\
lectores & 620 & 590 & 635 & 639 & 578 & 574 & 607 & 590 \\
Disléxicos & $(3.83)$ & $(3.92)$ & $(3.92)$ & $(3.83)$ & $(3.50)$ & $(1.67)$ & $(5.17)$ & $(3.33)$ \\
& & & & & & & & &
\end{tabular}

\begin{tabular}{lccccccccc}
\hline & \multicolumn{8}{c}{ Color de fondo verde } \\
\cline { 2 - 9 } & \multicolumn{1}{c}{ Global } & \multicolumn{7}{c}{ Local } \\
\cline { 2 - 9 } & \multicolumn{2}{c}{ Congruente } & \multicolumn{1}{c}{ Incongruente } & \multicolumn{2}{c}{ Congruente } & \multicolumn{2}{c}{ Incongruente } \\
\cline { 2 - 9 } & $\mathrm{C}$ & $\mathrm{U}$ & $\mathrm{C}$ & $\mathrm{U}$ & $\mathrm{C}$ & $\mathrm{U}$ & $\mathrm{C}$ & $\mathrm{U}$ \\
\hline Lectores & 644 & 633 & 693 & 669 & 677 & 637 & 692 & 671 \\
normales & $(3.75)$ & $(3.00)$ & $(4.67)$ & $(5.75)$ & $(4.00)$ & $(2.50)$ & $(3.75)$ & $(9.83)$ \\
Malos & 604 & 593 & 603 & 624 & 665 & 657 & 679 & 704 \\
lectores & $(13.42)$ & $(12.92)$ & $(17.42)$ & $(17.25)$ & $(8.08)$ & $(9.08)$ & $(16.08)$ & $(10.08)$ \\
Disléxicos & 593 & 557 & 631 & 595 & 575 & 581 & 607 & 592 \\
& $(1.00)$ & $(2.67)$ & $(3.75)$ & $(1.67)$ & $(4.58)$ & $(3.83)$ & $(4.25)$ & $(6.17)$ \\
& & & & & & & & \\
\hline
\end{tabular}

menores tasas de error (5.05) que los estímulos incongruentes (7.64). También resultaron significativas las interacciones entre condiciones de atención y grupo, $\mathrm{F}(2,33)=3.77, \mathrm{MCE}=484.34$, $\mathrm{p}=0.03$, congruencia y grupo $\mathrm{F}(2,33)=3.76$, $\mathrm{MCE}=207.45, \mathrm{p}=0.03$, así como la condición de color y atención, $\mathrm{F}(1,33)=4.90, \mathrm{MCE}=220.03$, $\mathrm{p}=0.03$.

La doble interacción entre condiciones de atención y grupo mostró que la tasa de errores en la condición de atención al nivel global era similar entre los sujetos normolectores (4.74) y disléxicos (3.07), mientras que los malos lectores mostraban una tasa de errores muy superior (13.70), que produjo diferencias significativas al compararlas con los otros dos grupos (diferencia entre malos lectores y normolectores = 8.96, $\mathrm{p}=0.02$; diferencia entre malos lectores $\mathrm{y}$ disléxicos $=10.63, \mathrm{p}<0.01)$. En la condición de atención al nivel local, los normolectores y los sujetos disléxicos también mostraron resultados similares (3.97 y 4.06 respectivamente), mientras que el grupo de malos lectores volvió a mostrar una tasa de errores (8.52) significativamente superior (diferencia entre malos lectores y normolectores $=4.55, \mathrm{p}=0.03$; diferencia entre malos lectores y disléxicos $=4.46, \mathrm{p}=0.03$ ). Como se puede ver en la Figura 2 a, las diferencias entre los grupos fueron más marcadas en la condición de atención al nivel global que en la de atención al nivel local.

La doble interacción entre congruencia y grupo (véase la Figura 2 b,) indicó que la tasa de errores en la condición congruente fue similar en los grupos de normolectores (3.36) y sujetos disléxicos (3.12), mientras que los malos lectores mostraron una tasa de errores (8.66) significativamente superior (diferencia entre malos 
a)

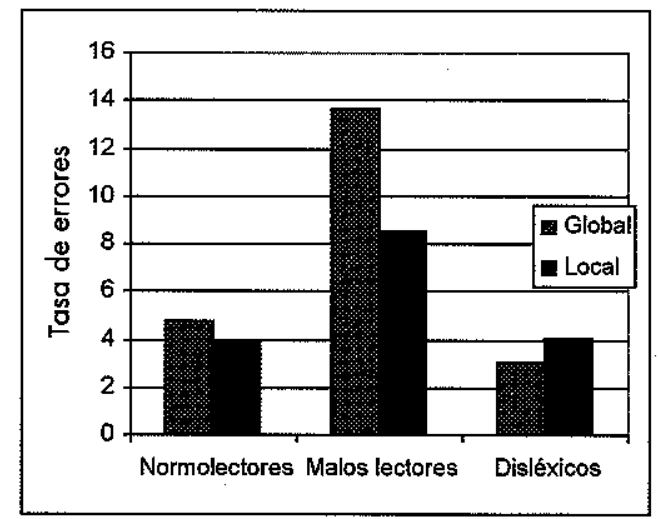

b)

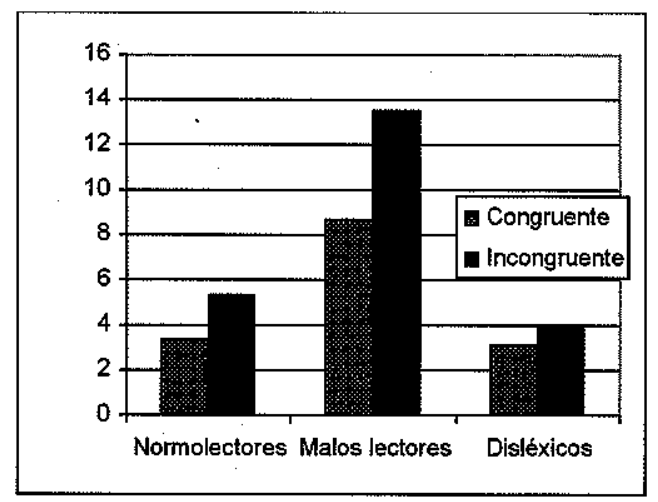

c)

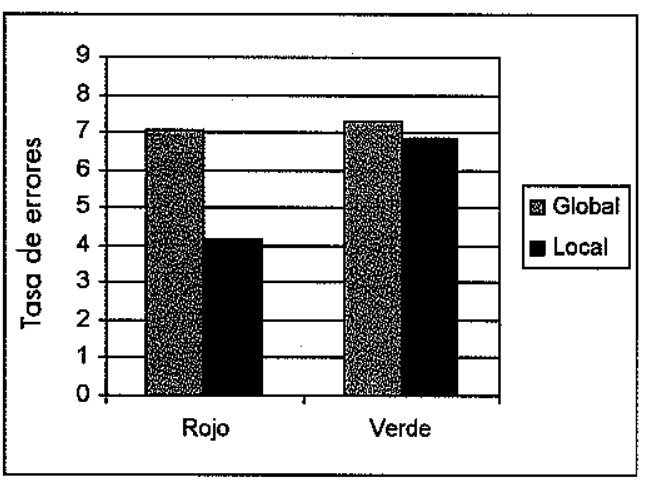

Figura 2. Interacciones significativas en las condiciones de: a) atención $x$ grupo, b) congruencia $x$ grupo y c) color $x$ atención. lectores y normolectores $=5.29, \mathrm{p}=0.04$; diferencia entre malos lectores y disléxicos $=5.53$, $\mathrm{p}=0.03$ ). La tasa de errores en la condición incongruente también fue significativamente distinta entre el grupo de malos lectores (13.56) y los otros dos grupos (normolectores $=5.34$, disléxicos $=4.01$; diferencia entre malos lectores y normolectores $=8.22, \mathrm{p}<0.01$; diferencia entre malos lectores y disléxicos $=9.55, \mathrm{p}<0.01$ ). Las diferencias fueron más marcadas en la condición de incongruencia que en la de congruencia.

La doble interacción entre condiciones de color y atención (véase la Figura 2 c), indicó que mientras que la tasa de errores en la condición de color de fondo verde fue similar en las dos condiciones de atención (global=7.27 y local $=6.85$ ), en la condición de color de fondo rojo, la tasa de errores en la condición de atención al nivel global (7.07) fue superior a la de la condición de atención al nivel local (4.18) lo que indica un efecto de primacía local.

\section{Discusión}

Los objetivos principales de este trabajo trataron de comprobar si los sujetos disléxicos presentaban déficits en el procesamiento global de patrones jerárquicos, que está mediado por el sistema magnocelular. Un segundo objetivo consistió en examinar los efectos del color de fondo (rojo y verde) en el procesamiento de la información global, y si el deterioro en la ejecución producida por el color de fondo rojo se producía únicamente en los sujetos que presentaban problemas en la lectura.

Los principales resultados de este trabajo indicaron que: a) los sujetos disléxicos no se diferenciaban de los lectores normales, en el procesamiento de la información global. Sin embargo, los malos lectores sí que se diferenciaron de manera significativa de los otros dos grupos; b) el color de fondo rojo empeoró el procesamiento de la información global y c) los efectos del color de fondo fueron similares en los tres grupos de sujetos.

Como se comentó en la Introducción, los resultados de Simmers y Bex (2001) mostraron que los sujetos disléxicos cometieron más errores en la formación de patrones globales (a partir de presentaciones locales) que los sujetos de 
control. Estos resultados coincidieron con los encontrados por Lewis y Frick (1999) según los cuales, muchos sujetos disléxicos presentaron dificultades de agrupamiento perceptivo de símbolos en filas. Sin embargo, los resultados del presente experimento muestran que eran los malos lectores y no los sujetos disléxicos los que se diferenciaron de los sujetos de control en el procesamiento de la información global. La discrepancia entre estos resultados y los hallados en trabajos anteriores (Lewis y Frick, 1999; Simmers y Bex, 2001) posiblemente se deba a las diferencias en la selección de los sujetos, porque en los trabajos previos no se tuvo en cuenta, de manera controlada, las puntuaciones en inteligencia de los sujetos y se pudieron seleccionar como sujetos disléxicos, sujetos malos lectores. Estos resultados son coherentes con los de Williams y Bologna (1095) y Sampascual (1990), que encontraron una relación inversa entre la eficacia lectora y agrupamiento perceptivo, que estaba acentuada en los malos lectores.

En cuanto a los efectos del color sobre el procesamiento de la información global y local, en nuestro trabajo se observó un deterioro de la precisión en el procesamiento de la información global cuando se utilizaron fondos de color rojo. Estos resultados son coherentes con los de Michimata et al. (1999), quienes propusieron que el sistema magnocelular era, en parte, responsable del efecto de precedencia global y que dicho sistema podía ser manipulado utilizando fondos de color rojo. En concreto, estos autores sugirieron que los resultados mostrados con un fondo rojo eran similares a los que se producirían si se atenuara el procesamiento de las bajas frecuencias espaciales. Esta teoría también puede explicar los resultados del presente trabajo porque si se disminuyera el procesamiento de las bajas frecuencias espaciales, los sujetos cometerían más errores cuando se presentan estímulos con mayor contenido en estas frecuencias (letras globales), mientras que los estímulos en los que fueran menos relevantes este tipo de frecuencias (estímulos locales) no sufrirían variaciones importantes. Como consecuencia, la diferencia en el número de errores entre los niveles global y local aumentaría; efecto que aparece en este experimento.

Por otro lado, los resultados no indicaron diferencias en los efectos del color de fondo entre unos grupos y otros, por lo que no se confirmó un deterioro en el sistema magnocelular en los sujetos con problemas de lectura.

En conclusión, estos resultados sugieren que las diferencias encontradas en trabajos anteriores, entre sujetos disléxicos y sujetos de control, en tareas de procesamiento visual podrían deberse a que no se igualaron los niveles de inteligencia de los grupos. El color de fondo rojo empeoró el procesamiento global, pero este efecto se dio en todos los grupos por igual, por lo que los resultados del presente estudio no apoyan la hipótesis de un déficit en el sistema magnocelular en sujetos con problemas de lectura.

\section{Referencias bibliográficas}

American Psychiatric Association (APA) (1994). Diagnostic and statistical manual of mental disorders $\left(4^{\circ} \mathrm{ed}\right.$.). Washington, D.C: APA.

Breitmeyer, B. (1980). Unmasking visual masking: a look at the "why" behind the veil of the "how". Psychology Review, 87, 52-69.

Breitmeyer, B.G. y Breier, J.I. (1994). Effects of background color on reaction time to stimuli varying in size and contrast: Inferences about human channels. Vision Research, 34, 1039-1045.

Breitmeyer, B.G. y Williams, M.C. (1990). Effects of isoluminant-background color on metacontrast and stroboscopic motion: Interactions between sustained $(\mathrm{P})$ and transient $(\mathrm{M})$ channels. Vision Research, 30, 1069-1075.

Chase, C.H. (1996). A visual deficit model of developmental dyslexia. En C.H. Chase, G.D. Rosen y G.F. Sherman (Eds.) Developmental dyslexia: neural, cognitive and genetic mechanisms, (pp.127-156). Timonium, MD: York Press.

Chase, C.H., Ashourzadeh, A., Kelly, C., Monfette, S. y Kinsey, K. (2003). Can the magnocellular pathway read? Evidence from studies of color. Vision Research, 43, 1211-1222.

Han, S. y Humphreys, G. (1999). Interactions between perceptual organization based on Gestalt laws and those based on hierarchical processing. Perception and Psychophysics, 61, 12871298.

Iovino, I., Fletcher, J.M., Breitmeyer, B.G. y Foorman, B.R. (1998). Colored overlays for visual perceptual deficits in children with reading disability and attention deficit/hyperactivity disorder: are they differentially effective? Journal of Clinical and Experimental Neuropsychology, 20 (6), 791-806. 
Lewis, J.P. y Frick, R.W. (1999). Row blindness in Gestalt grouping and developmental dyslexia. Neuropsychologia, 37, 385-393.

Livingstone, M.S. y Hubel, D.H. (1984). Anatomy and physiology of a color system in the primate visual cortex. Journal of Neuroscience, 4, 309356.

Livingstone, M.S., Rosen, G.D., Drislane, F.W. y Galaburda, A.M. (1991). Physiological and anatomical evidence for a magnocellular defect in developmental dyslexia. Proceedings of the National Academy of Sciences. USA. 88, 79437947.

Lovegrove, W.J. (1991). Spatial frecuency processing in dyslexic and normal readers. En J.F. Stein (Ed.) Vision and Visual Dyslexia: Vision and Visual dysfunction Vol 13, (pp. 148-153). CRC Press.

Marrocco, R.T., McClurkin, J.W. y Young, R.A. (1988). Spatial summation and conduction latency classification of cells in the lateral geniculate nucleus of macaques. Journal of Neuroscience, 2, 1275-1291.

Michimata, C., Okubo, M. y Mugishima, Y. (1999). Effects of background color on the global and local processing of hierarchically organized stimuli. Journal of Cognitive Neuroscience, 11 (1), 1-8.

Navon, D. (1977). Forest before trees: The precedence of global features in visual perception. Cognitive Psychology, 9, 353-383.

Ramus, F. (2001) Outstanding Questions about Phonological Processing in Dyslexia. Dyslexia, 7, 197-216.

Rutter, M. (1978). Prevalence and types of dyslexia. En A.L. Benton y D. Pearl, (Eds.) An Appraisal of
Current Knowledge (pp. 5-28). New York: Oxford University Press.

Sampascual, G. (1990). Atención selectiva en buenos y malos lectores. Revista de Psicología General y Aplicada, 43, 491-498.

Simmers, A.J. y Bex, P.J. (2001). Deficit of visual contour integration in dyslexia. Investigative Ophthalmology and Visual Science, 42 (11), 27372742 .

Skottun, B.C. (1997). The magnocellular deficit theory of dyslexia. Trends in Neuroscience, 20 (9), 397-398.

Skottun, B.C. (2000). The magnocellular deficit theory of dyslexia: the evidence from contrast sensitivity. Vision Research, 40, 111-127.

Skottun, B.C. y Parke, L.A. (1999). The possible relationship betwen visual deficits and dyslexia. Journal of Learning Disabilities, 32, 2-5.

Snow, C.E., Burns, M.S. y Griffin, P. (1998). Preventing Reading Difficulties in Young Children. Committee on the Prevention of Reading Difficulties in Young Children, National Research Council.

Solan, H.A. (1998). Influence of varying luminance and wavelength on comprehension and reading efficiency: a brief review of three studes. Journal of Optometric Vision Development, 29 (3), 98-103.

Tallal, P. (1980). Auditory temporal perception, phonics, and reading disabilities in children. Brain and Language, 9 (2), 182-198.

Williams, M.C. y Bologna, N.C.(1985). Perceptual grouping in good and poor readers. Perception and Psychophysics, 38, 367-374.

Yuste, C. (1995). Inteligencia general y factorial. Madrid: TEA Ediciones. 